\title{
Information Theoretic Analysis of LDS Scheme
}

\author{
Razavi. R, Student Member, IEEE, Hoshyar. R, Member, IEEE, Imran. M.A, Member, IEEE, Wang. Y, \\ Member, IEEE,
}

\begin{abstract}
In this paper, the capacity region of Low Density Signature Multiple Access Channel (LDS-MAC) is calculated through information theoretic analysis. LDS Code Division Multiple Access (LDS-CDMA) uses spreading sequences of low density for spreading the data symbols in time domain. This technique benefits from a less complex Multiuser Detector (MUD) compared to conventional CDMA with optimum MUD; while keeping the performance close to the single user scenario for up to $200 \%$ loaded conditions. Also evaluated is the effect of different factors on the capacity of LDS MAC.
\end{abstract}

Index Terms-Capacity, Low density signature, Multi-path fading channel

\section{INTRODUCTION}

$\mathbf{I}_{a}^{\mathrm{N}}$ $\mathrm{N}$ In multiuser detection the optimum maximum a posteriori (MAP) and Maximum Likelihood Multiuser Detectors (ML MUDs) [1],[2],[3] are capable of achieving the optimum BER performance that is close to the single-user BER bound. However, the complexity of these optimum MUDs is exponentially proportional to the number of users supported, which becomes extreme even when a moderate number of users are considered. Consequently, the application of the optimum MUDs in practice is limited.

Spreading sequences of the low-density type (containing many zeros) were theoretically presented in [4] for the purpose of allowing low-complexity multiuser detection. These sequences, when designed in accordance with suitable rules [5] allow application of a belief-propagation algorithm at the receiver. This was shown to result in promising performance for overloaded systems; i.e. systems with more spreading sequences than chips; and for Binary Phase Shift Keying (BPSK) modulated data in [6]. Recently, Low-Density Signature (LDS) Structure complemented with more-affordable MAP-based Chip-Level iterated (CLi) MUD has been shown to achieve performance very close to single-user even at $200 \%$ overloaded condition [6], [7]. The LDS structure can be captured by a low density graph thus the detection of different users' symbols could be based on Message Passing Algorithm (MPA) presented in [6] for LDS-CDMA systems.

In this contribution we investigate the information-theoretic

Manuscript received November 03, 2010. The associate editor coordinating the review of this letter and approving it for publication was P. Demestichas. This work was supported by Huawei Technologies. Co., Ltd, P.R.China.

R. Razavi and M. A. Imran are with the Centre for Communication Systems Research, University of Surrey, Guildford, Surrey, GU2 7XH, UK (e-mail: R.Razavi@surrey.ac.uk)

R. Hoshyar is with National Semiconductor, 2900 Semiconductor Drive PO Box 58090 Santa Clara, CA 95052-8090, United States

Y. Wang is with Huawei Technologies Co. Ltd, No.2222, Xin JinQiao Road, Pudong, Shanghai, 201206, P.R.China

Digital Object Identifier $\mathrm{xxxxx}$ capacity of LDS structure for uplink CDMA systems. Furthermore; using the calculated rate region, the effect of density factor and maximum number of allowed users per chip on the capacity of LDS MAC is investigated using Monte carlo simulations.

The rest of the paper is outlined as follows: Section II presents the system architecture for LDS scheme. In section III we derive the information-theoretic capacity of LDS structure. The simulation results for different LDS characteristics are presented in section IV. Conclusions are drawn in section $\mathrm{V}$.

\section{LDS SCHEME SYSTEM MODEL}

In LDS scheme, the low density spreading sequences are assigned uniquely to each user and are fully transparent to the receiver. So each user is intentionally arranged to spread its data over a small number of chips and then zero padding is done such that the processing gain stays the same as conventional CDMA systems. We focus on an LDS-CDMA system with $K$ users and user indices $k=1, \ldots, K$ and the multi access memoryless channel with probability transitions $p\left(y \mid x_{1}, \ldots, x_{K}\right)$. Input distribution $p\left(x_{1}\right) p\left(x_{2}\right) \ldots p\left(x_{K}\right)$ is considered independent.

Low density codebook with density factor $0<\zeta \leq 1$ is generated using the following steps.

1) Full density codebook generation: Generate the set of full density codewords (signatures) with length $\nu=\zeta N$ ( $N$ is the number of total chips or the length of the low density codewords) for each user $k$ according to its distribution $p\left(x_{k}\right)$.

2) Zero padding: Add $N-\nu$ zeros at the end of each codeword.

3) Interleaving: For each user randomly choose an interleaving pattern $\boldsymbol{\pi}_{k}=\left\{\pi_{k}(1), \pi_{k}(2), \ldots, \pi_{k}(N)\right\}$ and interleave the zero-padded codewords of the user. Thus the none zero components of codewords will appear in $\boldsymbol{\Omega}_{k}=\left\{\pi_{k}(1), \pi_{k}(2), \ldots, \pi_{k}(\nu)\right\}$ positions.

Let $\mathcal{P}=\left\{\boldsymbol{\pi}_{1}, \boldsymbol{\pi}_{2}, \ldots, \boldsymbol{\pi}_{K}\right\}$ denote the set of all users' interleavers. For a given time instance $i=1, \ldots, N$; let $K_{i}$ denote the set of users active at this time instance, i.e. $K_{i}=\left\{k \in K: i \in \Omega_{k}\right\}$. For any subset $S \subseteq K$ of users, we further define $\Omega(S)$ as the ordered set of time instances that users of $S$ are only active at that time, i.e. $\boldsymbol{\Omega}(S)=\left\{i=1, \ldots, N: S=K_{i}\right\}$. For a fixed density factor $\zeta$ as the overall codeword length approaches infinity, the length of the full density part will also approach to infinity. We can extend the joint typicality definition to low density codewords by applying the original definition over the support sets $\Omega(S)$ for every $S \subseteq K$. 
For time varying fading MAC we will have [8]:

$$
Y(i)=\sum_{k=1}^{K} H_{k}(i) X_{k}(i)+Z(i)
$$

where $i$ is the time index, $X_{k}(i)$ and $H_{k}(i)$ are the transmitted symbol and the fading process of user $k$ respectively, and $Z(i)$ is white Gaussian noise with variance $N_{0}$. So for LDS structure Eq.(1) can be modified as:

$$
Y(i)=\sum_{k \in K_{i}} H_{k}(i) X_{k}(i)+Z(i) .
$$

\section{CAPACITY REGION OF LDS MAC}

We shall now focus on the scenario of interest in this paper, where the channels are time-varying due to the motion of the users. When the receiver can perfectly track the channel but the transmitters don't have such information. So the codewords can not be selected as a function of the state of the channel but the decoding is able to use such information. The capacity region of time varying fading MAC is known (Gallager[9], Shamai and Wyner [10]) and given by:

$$
\begin{gathered}
\left\{\left(R_{1}, \ldots, R_{K}\right): \boldsymbol{R}(S) \leq \mathrm{E}_{\boldsymbol{H}}[\log (1+\right. \\
\left.\left.\left.\frac{\sum_{k \in S}\left|H_{k}\right|^{2} P_{k}}{N_{0}}\right)\right], \forall S \subseteq\{1, \ldots, K\}\right\}
\end{gathered}
$$

where $\boldsymbol{H}=\left(H_{1}, \ldots, H_{K}\right)$ is a random vector having the stationary distribution of the joint fading processes.

Now, the rate region of time varying fading LDS MAC can be derived by defining an equivalent LDS channel: $\tilde{H}_{k}(i)=$ $a_{k, i} H_{k}(i)$ where $a_{i, k}$ denotes the activity of user $k$ at time $i$, specifically $a_{k, i}$ will be equal to one if $k \in K_{i}$ otherwise it will be zero. The rate region of fading LDS MAC will be similar to original fading MAC but with the original channel replaced with LDS equivalent channel:

$$
\begin{aligned}
\left\{\left(R_{1}, \ldots, R_{K}\right): \boldsymbol{R}(S) \leq \mathrm{E}_{\tilde{H}}[\log (1+\right. \\
\left.\left.\left.\frac{\sum_{k \in S}\left|\tilde{H}_{k}\right|^{2} \tilde{P}_{k}}{N_{0}}\right)\right], \forall S \subseteq\{1, \ldots, K\}\right\}
\end{aligned}
$$

As for a proportion of time users are silent, their transmit power denoted by $\tilde{P}_{k}$ will be larger than their transmit power $\tilde{P}_{k}: P_{k} / \zeta$. More specifically, for a fixed interleaving pattern and thus a fixed LDS structure, the above rate region can be expressed as follows:

$$
\begin{gathered}
\left\{\left(R_{1}, \ldots, R_{K}\right): \boldsymbol{R}(S) \leq \frac{1}{N} \sum_{i=1}^{N} \mathrm{E}_{\boldsymbol{H}}[\log (1+\right. \\
\left.\left.\left.\frac{\sum_{k \in S} a_{k, i}\left|H_{k}\right|^{2} \tilde{P}_{k}}{N_{0}}\right)\right], \forall S \subseteq\{1, \ldots, K\}\right\}
\end{gathered}
$$

Now if we consider the ensemble of LDS schemes over all possible interleaving patterns, the activity parameter a will be independent and takes Bernoulli distribution with probability of being 1 equal to $\zeta$. Therefore the rate region will be:

$$
\begin{gathered}
\left\{\left(R_{1}, \ldots, R_{K}\right): \boldsymbol{R}(S) \leq \mathrm{E}_{\boldsymbol{a}, \boldsymbol{H}}[\log (1+\right. \\
\left.\left.\left.\frac{\sum_{k \in S} a_{k}\left|H_{k}\right|^{2} P_{k} / \zeta}{N_{0}}\right)\right], \forall S \subseteq\{1, \ldots, K\}\right\}
\end{gathered}
$$

where $\boldsymbol{a}=\left(a_{1}, \ldots, a_{K}\right) \in\{0,1\}^{K}$ with Bernoulli components with probability $\zeta$.

Bernoulli distribution of users' activity allows any number of users to be active in a given symbol. In this regard, we call the ensemble of the generated LDS codes as $\operatorname{LDS}_{K}(\zeta, K)$. If we limit the number of active users to be less than a specific number $\xi \leq K$, then the resulted LDS will be called $\operatorname{LDS}_{K}(\zeta, \xi)$. The rate region still will have the same form as above equation, but with a different distribution for activity vector $\boldsymbol{a}$. the original i.i.d Bernoulli distribution of the components of this vector will imply:

$$
p(\boldsymbol{a} ; \zeta, K)=\prod_{k=1}^{K} \zeta^{a_{k}}(1-\zeta)^{1-a_{k}}
$$

The distribution for $\operatorname{LDS}_{K}(\zeta, \xi)$ takes the following form:

$$
p(\boldsymbol{a} ; \zeta, \xi)= \begin{cases}\beta \prod_{k=1}^{K} \zeta^{a_{k}}(1-\zeta)^{1-a_{k}}, & \sum_{k=1}^{K} a_{k} \leq \xi \\ 0, & \text { otherwise }\end{cases}
$$

where $\beta$ is a normalizing factor and is expressed as follows:

$$
\beta=\left(\sum_{d=0}^{\xi}\left(\begin{array}{l}
K \\
d
\end{array}\right) \zeta^{d}(1-\zeta)^{K-d}\right)^{-1}
$$

Clearly having a symbol with no active user is a waist of channel. We can further restrict the LDS ensemble to those that have at least one user active at a given time. This will result in the expurgated scheme $\operatorname{LDS}_{K}\left(\zeta, \xi^{+}\right)$with following activity vector distribution:

$$
\begin{aligned}
p\left(\boldsymbol{a} ; \zeta, \xi^{+}\right) & = \\
& \begin{cases}\beta^{+} \prod_{k=1}^{K} \zeta^{a_{k}}(1-\zeta)^{1-a_{k}}, & 0<\sum_{k=1}^{K} a_{k} \leq \xi \\
0, & \text { otherwise }\end{cases}
\end{aligned}
$$

where

$$
\beta^{+}=\left(\sum_{d=1}^{\xi}\left(\begin{array}{l}
K \\
d
\end{array}\right) \zeta^{d}(1-\zeta)^{K-d}\right)^{-1}
$$

It is noticeable that by introducing the constraint of maximum number of active users at a specific time instance, the density factor $\zeta$ would diverge from its initial value $\zeta_{0}$ according to number of active users (d). The $\zeta$ can be calculated as follows:

$$
\zeta=\sum_{d=1}^{\xi} p(d) \zeta(d)
$$




$$
\begin{aligned}
p(d)= & \left(\begin{array}{l}
K \\
d
\end{array}\right)\left(1-\zeta_{0}\right)^{K-d} \zeta_{0}^{d} \beta^{+} \quad \& \quad \zeta(d)=\frac{d}{K}, \\
& \Rightarrow \zeta=\sum_{d=1}^{\xi}\left(\begin{array}{l}
K \\
d
\end{array}\right) \zeta_{0}^{d}\left(1-\zeta_{0}\right)^{K-d} \beta^{+} \frac{d}{K} .
\end{aligned}
$$

By plugging density factor $\zeta$ from Eq. (14) into Eq. (6) we obtain the capacity region of LDS MAC.

\section{Numerical Results}

In this section, we present the simulation results for both MAC and LDS MAC systems. The simulation is carried out for a system with 20 users. the Weighted Sum Rate (WSR) is calculated for LDS fading MAC by averaging over fading process through Monte Carlo simulation. The results for MAC are calculated using Eq. (3) for 20 users.

Fig. 1 shows the comparison between the WSR of MAC and LDS MAC for different density factors and maximum number of users allowed per chip. As expected there is a gap between the curve for MAC and LDS MAC, which is the penalty paid for reducing the complexity of multiuser detector compared to successive decoding for achieving the points on the boundary of MAC capacity region. It is noticeable that for LDS systems increasing $\zeta$ and reducing the maximum number of allowed users at each time instance will result in higher WSR.

Using LDS structure the complexity order of the MUD will reduce from number of total users' to the maximum number of allowed users per chip as shown in Fig. 2 This figure shows that by reducing the density factor, the computational complexity of MUD for LDS systems decreases significantly.

\section{CONCLUSIONS}

The capacity region of LDS MAC is calculated using information theoretic analysis. It is shown that using LDS as a multiple access technique will reduce the capacity compared to MAC with successive decoding, but this can be interpreted as the price paid for reducing the complexity of receiver. Furthermore the simulation results show how the capacity is changed according to density factor and maximum number of users at each time instance. As expected as the density factor is increased, the capacity approaches to that of MAC.

\section{REFERENCES}

[1] S. Verdu, "Minimum probability of error for asynchronous multiple access communication systems," in Proc. IEEE Military Communications Conf. MILCOM, vol. 1, 1983, pp. 213-219.

[2] - "Minimum probability of error for asynchronous gaussian multiple-access channels," IEEE Transactions on Information Theory, vol. 32, no. 1, pp. 85-96, 1986.

[3] _ Multiuser Detection. Cambridge University Press, 1998.

[4] J. Choi, "Low density spreading for multicarrier systems," in Proc. IEEE Eighth Int Spread Spectrum Techniques and Applications Symp, 2004, pp. 575-578.

[5] A. Montanari and D. Tse, "Analysis of belief propagation for non-linear problems: The example of CDMA (or: How to prove Tanaka's formula)," in Proc. ITW '06 Punta del Este Information Theory Workshop IEEE, 2006, pp. $160-164$.

[6] R. Hoshyar, F. P. Wathan, and R. Tafazolli, "Novel low-density signature for synchronous CDMA systems over AWGN channel," IEEE Trans. Signal Processing, vol. 56, no. 4, pp. 1616-1626, 2008.

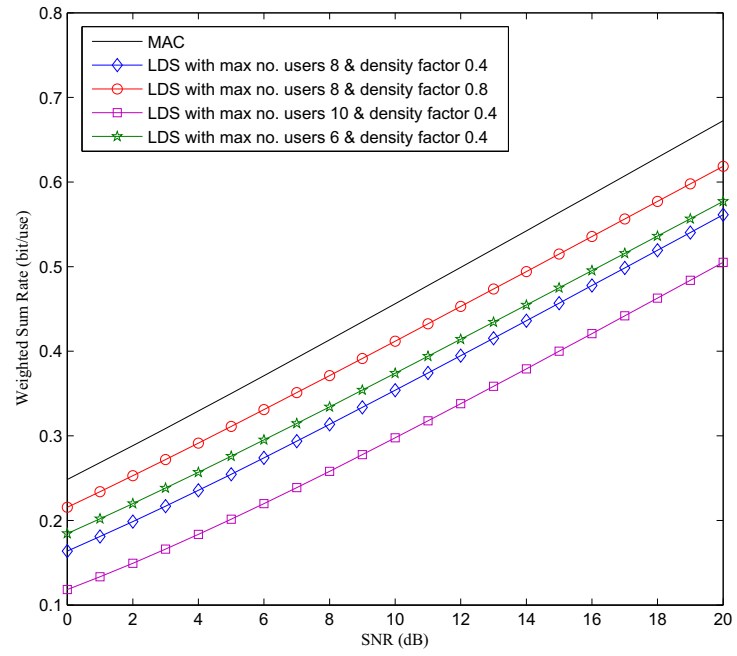

Fig. 1. Weighted sum rate for different density factors and maximum number of allowed users per chip.

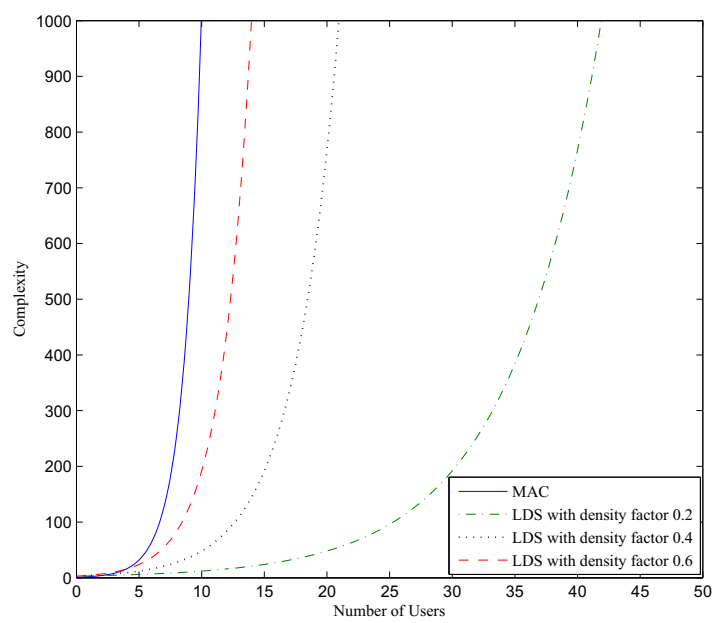

Fig. 2. Complexity of MUD for MAC in comparison with LDS with different density factors.

[7] R. Hoshyar, R. Razavi, and M. Al-Imari, "LDS-OFDM an efficient multiple access technique," in Proc. IEEE 71st Vehicular Technology Conf. (VTC 2010-Spring), 2010, pp. 1-5.

[8] D. N. C. Tse and S. V. Hanly, "Multiaccess fading channels. i. polymatroid structure, optimal resource allocation and throughput capacities," IEEE Trans. Information Theory, vol. 44, no. 7, pp. 2796-2815, 1998.

[9] R. Gallager, "An inequality on the capacity region of multiaccess fading channels," Communications and CryptographyTwo Sides of One Tapestry, p. 129139, 1994.

[10] S. Shamai and A. D. Wyner, "Information-theoretic considerations for symmetric, cellular, multiple-access fading channels. ii," IEEE Trans. Information Theory, vol. 43, no. 6, pp. 1895-1911, 1997. 